\title{
REPRODUCING KERNEL HILBERT SPACES GENERATED BY THE BINOMIAL COEFFICIENTS
}

\author{
DANIEL ALPAY AND PALLE JORGENSEN
}

\begin{abstract}
We study a reproducing kernel Hilbert space of functions defined on the positive integers and associated to the binomial coefficients. We introduce two transforms, which allow us to develop a related harmonic analysis in this Hilbert space. Finally, we mention connections with the theory of discrete analytic functions, statistics, and with the quantum case.
\end{abstract}

\section{Contents}

1. Introduction

2. Some formulas for binomial coefficients

3. The binomial Fourier transform

4. The second transform

5. Motivation from discrete analytic functions the

6. The quantum case

7. A one parameter group of transforms

References

\section{INTRODUCTION}

We show that the binomial coefficients form an orthonormal basis (ONB) in a naturally formed reproducing kernel Hilbert space $\mathcal{H}(K)$. We find explicit properties and formulas for the kernel, and we identify two pairs of transforms, again with explicit formulas. With the binomial functions forming an ONB in $\mathcal{H}(K)$, one naturally then gets transforms as isometric isomorphisms between

1991 Mathematics Subject Classification. 46E22, 47H60, $11 \mathrm{~B} 65$.

Key words and phrases. Binomial Fourier transform, binomial coefficients, reproducing kernel, Hilbert space, Hardy space, positive definite, multipliers, infinite matrices, Hurwitz series, discrete analytic functions.

D. Alpay thanks the Earl Katz family for endowing the chair which supported his research. The research of the authors was supported in part by the Binational Science Foundation grant 2010117. 
$\mathcal{H}(K)$ and $\ell^{2}\left(\mathbb{Z}_{+}\right)$. We write down two such transforms, and for each we compute its inverse. Our work is motivated by applications to discrete analytic functions [2]. This is followed up here, and we outline additional applications to combinatorial probability theory. In 2, we introduce a product (in the sense of hypergroups [13]) in spaces of discrete analytic functions. We use our present transforms for computing this product. In the last section, as a corollary to Theorem 7.3 we establish a significant statistical property for our one-parameter family of kernels $K_{\lambda}$, as well as for the corresponding spectral transforms: we show for each kernel within our family of kernels $K_{\lambda}$ there is a probability space; hence an associated indexed family of probability spaces $\left(\Omega, \mathcal{F}, P^{\lambda}\right)$. We prove that as the value of the parameter $\lambda$ vary, the corresponding measures $P^{\lambda}$ are mutually singular. For readers not familiar with reproducing kernels, we suggest [1, 3, 16, 18, 19]. For a sample of references to combinatorial probability, see e.g, [14, 15, 21].

Motivated by our previous work on discrete analytic functions [2] we study a reproducing kernel space of functions from $\mathbb{Z}_{+}=\{0,1,2, \ldots\}$ into $\mathbb{R}$ associated to the binomial coefficients.

We study the reproducing kernel Hilbert space (RKHS) $\mathcal{H}(K)$ generated by the kernel $K(x, y)$ consisting of the numbers in the Pascal triangle, i.e., for every $(x, y)$ in $\mathbb{Z}_{+} \times \mathbb{Z}_{+}$, set $K(x, y)=\left(\begin{array}{c}x+y \\ x\end{array}\right)$. Using the Chu-Vandermonde formula, one sees that $K(x, y)$ is positive definite in the sense of kernel theory, hence the RKHS. We study a number of analytic features of $\mathcal{H}(K)$ : We find families of orthonormal bases in $\mathcal{H}(K)$, and we prove associated transform theorems. Moreover we compute these transforms explicitly, making use of infinite square matrices of upper and lower triangular form. We further show that our transforms facilitate analysis on $\mathcal{H}(K)$. We further sketch a oneparameter deformation family with application .

In the remaining part of this section we give results characterizing vectors in their respective reproducing kernel Hilbert spaces; see Theorem 1.1

Definitions. Let for $x, n \in \mathbb{Z}_{+}$such that $n \leq x$

$$
\left(\begin{array}{l}
x \\
n
\end{array}\right)=\frac{x(x-1) \cdots(x-n+1)}{n !}
$$

the binomial coefficient. We set

$$
e_{n}(x)= \begin{cases}0, & \text { if } \quad x<n, \\
\left(\begin{array}{l}
x \\
n
\end{array}\right)=\frac{x(x-1) \cdots(x-n+1)}{n !}, & \text { if } \quad x \geq n,\end{cases}
$$


and

$$
K(x, y)=\sum_{n=0}^{\infty} e_{n}(x) e_{n}(y)=\left(\begin{array}{c}
x+y \\
x
\end{array}\right) \quad x, y \in \mathbb{Z}_{+} .
$$

The sum (1.3) is always finite and well defined, and defines a positive definite function on $\mathbb{Z}_{+}$.

Using the Chu-Vandermonde formula (see for instance [20, formula (10), p. 217])

$$
\left(\begin{array}{c}
x+y \\
t
\end{array}\right)=\sum_{k=0}^{t}\left(\begin{array}{l}
x \\
k
\end{array}\right)\left(\begin{array}{c}
y \\
t-k
\end{array}\right)
$$

(with $t, x, y \in \mathbb{N}_{0}$ and $t \leq y$ ) one can rewrite $K(x, y)$ as

$$
K(x, y)=\sum_{n=0}^{x \wedge y}\left(\begin{array}{l}
x \\
n
\end{array}\right)\left(\begin{array}{l}
y \\
n
\end{array}\right)
$$

or, equivalently,

$$
K(x, y)=\sum_{n=0}^{x \wedge y} \frac{x^{[n]} y^{[n]}}{(n !)^{2}}
$$

where we have set

$$
x^{[n]}=x(x-1) \cdots(x-n+1) .
$$

Writing the kernel $K(x, y)$ as an infinite matrix $K$ we note that we have

$$
K=L L^{*}
$$

where $L$ denote the following infinite lower triangular matrix consisting of the binomial coefficients

(1.9)

$L=$

$$
=\left(\begin{array}{cccccccccccc}
1 & 0 & 0 & 0 & 0 & 0 & 0 & \cdots & . & 0 & \cdots \\
1 & 1 & 0 & 0 & 0 & 0 & 0 & \cdots & . & 0 & \cdots \\
1 & 2 & 1 & 0 & 0 & 0 & 0 & \cdots & . & 0 & \cdots \\
1 & 3 & 3 & 1 & 0 & 0 & 0 & \cdots & . & 0 & \cdots \\
1 & 4 & 6 & 4 & 1 & 0 & 0 & \cdots & & . & 0 & \cdots \\
\vdots & \vdots & & & & & & & & & \\
1 & n & \left(\begin{array}{c}
n \\
2
\end{array}\right) & \cdots & \left(\begin{array}{c}
n \\
2
\end{array}\right) & n & 1 & 0 & 0 & \cdots & 0 & \cdots \\
1 & n+1 & \left(\begin{array}{c}
n+1 \\
2
\end{array}\right) & \ldots & & \left(\begin{array}{c}
n+1 \\
2
\end{array}\right) & n+1 & 1 & 0 & \cdots & 0 & \cdots \\
\vdots & \vdots & \vdots & & & \vdots & \vdots & 1 & & & \\
\vdots & \vdots & \vdots & & & \vdots & \vdots & \vdots & \vdots & & &
\end{array}\right)
$$


We denote by $\mathcal{H}(K)$ the reproducing kernel of real valued functions with domain $\mathbb{Z}_{+}$and with reproducing kernel $K(x, y)$. This latter space consists of all functions of the form

$$
g(x)=\sum_{n=0}^{\infty} a_{n} e_{n}(x)
$$

where the $a_{n} \in \mathbb{R}$, and with norm

$$
\|g\|_{\mathcal{H}(K)}^{2}=\sum_{n=0}^{\infty} a_{n}^{2}
$$

See [19, corollary 4, p. 169].

It follows from (1.8) that we have:

Theorem 1.1. Let $f \in \mathbb{R}^{\mathbb{Z}_{+}}$. Then,

$$
\sum_{x \in \mathbb{Z}_{+}} K(\cdot, x) f(x) \in \mathcal{H}(K) \quad \Longleftrightarrow \quad L^{*} f \in \ell^{2}\left(\mathbb{Z}_{+}\right) .
$$

Organization. The paper consists of seven sections and of an appendix besides the introduction, and its outline is as follows: In Section 2 we review some results on binomial coefficients. Sections 3, 4 contain the main results of the paper, on the above mentioned transforms. The first transform, which we call the binomial Fourier transform, and its inverse, are studied in Section 3. A natural isomorphism between $\mathcal{H}(K)$ and the Hardy space $\mathbf{H}^{2}(\mathbb{D})$ is defined in that section. The following two sections, Section 4 is devoted to the second transform. In Section 5 we study some links with the theory of discrete analytic functions. In Section [6 we considered the case of $q$-binomial coefficients. We present some applications in the last section.

\section{SOME FORMULAS FOR BINOMIAL COEFFICIENTS}

In this section we establish some duality relations for the binomial functions in (1.2), beginning with Lemma 3.1. This lemma in turn will be used in our results from sections 3 through 4 dealing with the two transforms. The transforms throw light on the RKHS of the binomial functions, but they also imply new formulas for these functions, linear relations (Theorem 3.3), and their use in the study of discrete analytic functions (section 5).

We make the following summation convention. In computations below, we will be using summations over index-values in finite or infinite subsets of $\mathbb{Z}_{+}=\{0,1,2, \cdots\} ;$ in some cases, multiple summations inside a single computation. These summations will then always entail ranges over summation indices $x, y, k, n, \cdots$ which are limited by choice of segments in $\mathbb{Z}_{+}$; and the respective summations will be specified by the segment-endpoints. 
Lemma 2.1. Let $m, n \in \mathbb{Z}_{+}$be such that $m \leq n$. Then,

$$
\sum_{j=m}^{n}(-1)^{m+j}\left(\begin{array}{c}
n \\
j
\end{array}\right)\left(\begin{array}{c}
j \\
m
\end{array}\right)=\delta_{m, n}
$$

Proof. The result is clear when $m=n$. Let us assume now $m<n$. We first note that

$$
\begin{aligned}
\left(\begin{array}{c}
n \\
j
\end{array}\right)\left(\begin{array}{c}
j \\
m
\end{array}\right) & =\frac{n !}{(n-j) ! j !} \frac{j !}{(j-m) ! m !} \\
& =\frac{n !}{(n-j) !(j-m) ! m !} \\
& =\frac{n !}{(n-m) ! m !} \frac{(n-m) !}{(n-j) !(j-m) !} \\
& =\left(\begin{array}{c}
n \\
m
\end{array}\right)\left(\begin{array}{c}
n-m \\
j-m
\end{array}\right) .
\end{aligned}
$$

Hence,

$$
\begin{aligned}
\sum_{j=m}^{n}(-1)^{m+j}\left(\begin{array}{l}
n \\
j
\end{array}\right)\left(\begin{array}{c}
j \\
m
\end{array}\right) & =\sum_{j=m}^{n}(-1)^{m+j}\left(\begin{array}{c}
n \\
m
\end{array}\right)\left(\begin{array}{c}
n-m \\
j-m
\end{array}\right) \\
& =\left(\begin{array}{c}
n \\
m
\end{array}\right) \sum_{j=m}^{n}(-1)^{m+j}\left(\begin{array}{c}
n-m \\
j-m
\end{array}\right)
\end{aligned}
$$

and setting $\ell=j-m$,

$$
\begin{aligned}
& =\left(\begin{array}{c}
n \\
m
\end{array}\right) \sum_{\ell=0}^{n-m}(-1)^{\ell}\left(\begin{array}{c}
n-m \\
\ell
\end{array}\right) \\
& =\left(\begin{array}{c}
n \\
m
\end{array}\right)(1-1)^{n-m} \\
& =0 .
\end{aligned}
$$

The following result is a corollary of Lemma 2.1, and plays an important

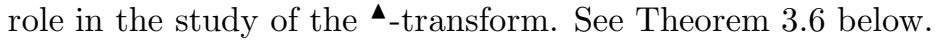

Theorem 2.2. Let $K(\ell, m)$ be defined by (1.5). Then it holds that:

$$
\sum_{\ell=0}^{n}(-1)^{n+\ell}\left(\begin{array}{l}
n \\
\ell
\end{array}\right) K(\ell, m)= \begin{cases}\left(\begin{array}{l}
m \\
n
\end{array}\right), & \text { if } \quad m \geq n, \\
0, & \text { if } \quad m<n .\end{cases}
$$


Proof. By the definition of $K$, we have

$$
\begin{aligned}
\sum_{\ell=0}^{n}(-1)^{n+\ell}\left(\begin{array}{c}
n \\
\ell
\end{array}\right) K(\ell, m) & =\sum_{\ell=0}^{n} \sum_{j=0}^{m \wedge \ell}(-1)^{n+\ell}\left(\begin{array}{c}
n \\
\ell
\end{array}\right)\left(\begin{array}{c}
m \\
j
\end{array}\right)\left(\begin{array}{l}
\ell \\
j
\end{array}\right) \\
& =\sum_{j=0}^{n \wedge m}\left(\begin{array}{c}
m \\
j
\end{array}\right)\left(\sum_{\ell=j}^{n}(-1)^{n+\ell}\left(\begin{array}{l}
n \\
\ell
\end{array}\right)\left(\begin{array}{l}
\ell \\
j
\end{array}\right)\right)
\end{aligned}
$$

and, applying Lemma 2.1

$$
\begin{aligned}
& =\sum_{j=0}^{n \wedge m}\left(\begin{array}{c}
m \\
j
\end{array}\right) \delta_{j, n} \\
& =\left\{\begin{array}{l}
\left(\begin{array}{l}
m \\
n
\end{array}\right), \quad \text { if } \quad m \geq n, \\
0, \quad \text { if } \quad m<n .
\end{array}\right.
\end{aligned}
$$

Lemma 2.3. Let $K(x, y)$ be defined by (1.5). Then it holds that:

$$
\begin{aligned}
K(x, x) & =\sum_{n=0}^{x}\left(\begin{array}{l}
x \\
n
\end{array}\right)^{2}=\left(\begin{array}{c}
2 x \\
x
\end{array}\right)=e_{x}(2 x), \\
|K(x, y)| & \leq K(x, x)^{1 / 2} K(y, y)^{1 / 2}=\left(\left(\begin{array}{c}
2 x \\
x
\end{array}\right)\left(\begin{array}{c}
2 y \\
y
\end{array}\right)\right)^{1 / 2} .
\end{aligned}
$$

Proof. The first claim follows from setting $x=y$ in (1.5). The second claim follows from the Cauchy-Schwarz inequality, since $K$ is positive definite.

\section{The Binomial Fourier transform}

In this section we introduce the first family of transforms. We then use them in order to characterize functions on $\mathbb{Z}_{+}$belonging to $\mathcal{H}(K)$, their properties, both analytic and algebraic. For example in Theorem 3.3, we give a natural isomorphism between $\mathcal{H}(K)$ and the Hardy space of the disk. In Theorem 3.14 we show that the orthonornal vectors $e_{n}$ in $\mathcal{H}(K)$ from (1.2) generate an algebra, or rather a hypergroup. See [13.

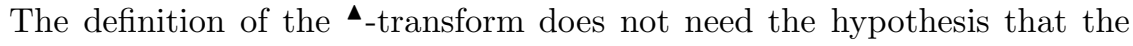
sequence is in $\ell_{2}\left(\mathbb{Z}_{+}\right)$, and is as follows: 
Definition 3.1. Let $f$ be a function from $\mathbb{Z}_{+}$into $\mathbb{R}$. The binomial Fourier transform of $f$ is defined by

$$
f^{\mathbf{\Lambda}}(x)=\sum_{j=0}^{x}\left(\begin{array}{l}
x \\
j
\end{array}\right) f(j), \quad x=0,1,2 \ldots
$$

Thus,

$$
\begin{aligned}
f^{\boldsymbol{\Delta}}(x) & =f(0)+x f(1)+\frac{x(x-1)}{2} f(2)+\frac{x(x-1)(x-2)}{6} f(3)+\cdots \\
& =\sum_{n=0}^{\infty} f(n) e_{n}(x) .
\end{aligned}
$$

The sums in (3.1) are finite, and $f^{\boldsymbol{\Lambda}}$ exists for every function from $\mathbb{Z}_{+}$into $\mathbb{R}$. We note that (3.1) can be written as

$$
f^{\mathbf{\Delta}}=L f=L(1) f
$$

where $L(\lambda)$ is defined in Section 7 below by (7.3).

The image of the function $f(j) \equiv 0$ is the zero function. The image of $f(j)=(-1)^{j}$ is the function

$$
f^{\boldsymbol{\Lambda}}(x)=\delta_{0, x}
$$

while the image of $f(j)=1$ is $f^{\mathbf{\Delta}}(x)=2^{x}$.

Another example of interest is:

Example 3.2. Let $f_{a}(j)=a^{* j}$, where $a \in \mathbb{C}$. Then,

$$
f^{\mathbf{\Lambda}}(x)=\sum_{n=0}^{\infty} a^{* n} e_{n}(x)
$$

is given by

$$
f_{a}^{\mathbf{\Delta}}(x)=\sum_{j=0}^{x}\left(\begin{array}{l}
x \\
j
\end{array}\right) a^{* j}=\left(1+a^{*}\right)^{x}, \quad x=0,1,2, \ldots
$$

Let now $\mathbf{H}^{2}(\mathbb{D})$ denote the Hardy space of complex-valued functions $f(z)=$ $\sum_{n=0}^{\infty} a_{n} z^{n}$ analytic in the open unit disk and such that

$$
\|f\|_{2}^{2}:=\sum_{n=0}^{\infty}\left|a_{n}\right|^{2}<\infty .
$$

We define a map $\psi$ from the complexified of $\mathcal{H}(K)$ into $\mathbf{H}^{2}(\mathbb{D})$ by

$$
\psi\left(e_{n}\right)=z^{n} .
$$


Theorem 3.3. The map $\psi$ is unitary from the complexified of $\mathcal{H}(K)$ onto the Hardy space $\mathbf{H}^{2}(\mathbb{D})$. Moreover it associates to the function $f_{a}: x \mapsto$ $\left(1+a^{*}\right)^{x}, x \in \mathbb{Z}_{+}$the function $z \mapsto \frac{1}{1-z a^{*}}, z \in \mathbb{D}$. In particular, for $a, b$ in the open unit disk $\mathbb{D}$

$$
\left\langle f_{a}^{\mathbf{\Delta}}, f_{b}^{\mathbf{\Delta}}\right\rangle_{\mathcal{H}(K)}=\frac{1}{1-a^{*} b} .
$$

Proof. The map is unitary since it maps an orthonormal basis onto an orthonormal basis. The claim on the image of the sequence $f_{a}$ follows from (3.6) and (3.5). Finally, (3.9) follows from the unitarity of $\psi$, the formula

$$
\frac{1}{1-z a^{*}}=\sum_{n=0}^{\infty} z^{n} a^{* n}
$$

and the definition of the inner product of $\mathbf{H}^{2}(\mathbb{D})$.

The next proposition is used to prove that the map $\mathbf{\Delta}$ is unitary from $\ell^{2}\left(\mathbb{Z}_{+}\right)$ onto $\mathcal{H}(K)$. See Theorem 3.6 .

Proposition 3.4. Let $e_{n}$ defined by (1.2), and let

$$
\widetilde{e_{n}}(m)=(-1)^{n+m} e_{n}(m), \text { and } \quad \delta_{n}(m)=\delta_{n, m} .
$$

Then

$$
\delta_{n}^{\mathbf{\Lambda}}=e_{n} \quad \text { and } \quad\left(\widetilde{e_{n}}\right)^{\mathbf{\Lambda}}=\delta_{n} .
$$

Proof. The first claim follows from the definition of $e_{n}$. As for the second claim, we have:

$$
\begin{aligned}
\left(\widetilde{e_{n}}\right)^{\mathbf{\Lambda}}(m) & =\sum_{j=0}^{m}\left(\begin{array}{c}
m \\
j
\end{array}\right)(-1)^{n+j} e_{n}(j) \\
& =\left\{\begin{array}{l}
0, \quad \text { if } \quad m<n \quad\left(\text { since } e_{n}(j)=0 \text { for } j<n\right) \\
\sum_{j=n}^{m}\left(\begin{array}{c}
m \\
j
\end{array}\right)\left(\begin{array}{l}
j \\
n
\end{array}\right)(-1)^{n+j}, \quad \text { if } \quad m \geq n .
\end{array}\right.
\end{aligned}
$$

We conclude by using Lemma 2.1 to compute this last sum.

It follows from (3.9) that most, if not all, the problems considered in $\mathbf{H}^{2}(\mathbb{D})$ can be transposed in a natural way in the space $\mathcal{H}(K)$.

We now define the function $\epsilon_{n}(j)=e_{j}(n)$. Thus $\left(\epsilon_{n}(j)\right)$ can be identified with the finitely nonzero sequence of $\ell^{2}\left(\mathbb{Z}_{+}\right)$whose nonzero terms are the first $n+1$ terms given by $\left(\begin{array}{c}n \\ j\end{array}\right), j=0, \ldots, n$. 
Lemma 3.5. Let $n, m \in \mathbb{Z}_{+}$. Then,

$$
\begin{aligned}
\left(\epsilon_{n}^{\mathbf{\Delta}}\right)(x) & =K(x, n) \\
\left\langle\epsilon_{n}, \epsilon_{m}\right\rangle_{\ell^{2}\left(\mathbb{Z}_{+}\right)} & =\langle K(\cdot, n), K(\cdot, m)\rangle_{\mathcal{H}(K)} .
\end{aligned}
$$

Proof. By definition of the transform,

$$
\left(\epsilon_{n}^{\mathbf{\Delta}}\right)(x)=\sum_{j=0}^{x}\left(\begin{array}{l}
x \\
j
\end{array}\right) \epsilon_{n}(j)=\sum_{j=0}^{x \wedge n}\left(\begin{array}{l}
x \\
j
\end{array}\right)\left(\begin{array}{l}
n \\
j
\end{array}\right)=K(x, n),
$$

and so (3.13) holds. To prove (3.14), it suffices to notice that

$$
\left\langle\epsilon_{n}, \epsilon_{m}\right\rangle_{\ell^{2}\left(\mathbb{Z}_{+}\right)}=\sum_{j=0}^{n \wedge m}\left(\begin{array}{c}
n \\
j
\end{array}\right)\left(\begin{array}{c}
m \\
j
\end{array}\right)=K(n, m) .
$$

Theorem 3.6. The map $f \mapsto f^{\boldsymbol{\Lambda}}$ defines a unitary mapping from $\ell_{2}\left(\mathbb{Z}_{+}\right)$ onto $\mathcal{H}(K)$, and its inverse is given by either formulas

$$
g^{\mathbf{V}}=\sum_{n=0}^{\infty} a_{n}(-1)^{n} \delta_{n} \quad \text { for } \quad g=\sum_{n=0}^{\infty} a_{n} e_{n} \in \mathcal{H}(K),
$$

or

$$
g^{\mathbf{V}}(n)=\sum_{\ell=0}^{n}\left(\begin{array}{l}
n \\
\ell
\end{array}\right) g(\ell)(-1)^{\ell-n} .
$$

We note that (3.18) can be rewritten as

$$
g^{\boldsymbol{\nabla}}=L(-1) g,
$$

where $L(\lambda)$ is defined by (7.3) below.

Proof of Theorem 3.6. We present two proofs. The first is based on Proposition 3.4. Let

$$
f=\sum_{n=0}^{N} a_{n} \delta_{n}
$$

be a real valued function on $\mathbb{Z}_{+}$with finite support. Then,

$$
f^{\mathbf{\Lambda}}=\sum_{n=0}^{N} a_{n} e_{n} \in \mathcal{H}(K)
$$

and

$$
\left\|f^{\boldsymbol{\Lambda}}\right\|_{\mathcal{H}(K)}^{2}=\|f\|_{\ell^{2}\left(\mathbb{Z}_{+}\right)}^{2}=\sum_{n=0}^{N} a_{n}^{2} .
$$


The result follows by taking limits.

The second proof uses Lemma 3.5. The functions $\epsilon_{0}, \epsilon_{1}, \ldots$ span a dense set of $\ell_{2}\left(\mathbb{Z}_{+}\right)$, and therefore (3.14) extends to an isometry. The isometry is onto since the kernels span a dense set of $\mathcal{H}(K)$. Therefore the transform $f^{\mathbf{\Lambda}}$ is unitary. To prove its inverse is given by (3.18) we note that (2.4) can be rewritten as

$$
\left(K_{m}^{\mathbf{\nabla}}\right)(n)=\epsilon_{m}(n), \quad n \in \mathbb{Z}_{+} .
$$

As a corollary of the above, the next results offer an answer to the following question: Given a function $f$ on $\mathbb{Z}_{+}$, when does $f$ belong to the reproducing kernel Hilbert space $\mathcal{H}(K)$ with reproducing kernel (1.5)?

Corollary 3.7. Let $f: \mathbb{Z}_{+} \longrightarrow \mathbb{R}$ be a function, and set

$$
f^{\mathbf{\nabla}}(x)=\sum_{\ell=0}^{x}\left(\begin{array}{l}
x \\
\ell
\end{array}\right) f(\ell)(-1)^{\ell-x} .
$$

Then, $f \in \mathcal{H}(K)$ if and only if the transform $x \mapsto f^{\mathbf{v}}(x)$ is in $\ell^{2}\left(\mathbb{Z}_{+}\right)$.

Proof. In Theorem 3.6 we proved that if $f \in \mathcal{H}(K)$ then its transform $f^{\boldsymbol{\nabla}}$ belongs to $\ell^{2}\left(\mathbb{Z}_{+}\right)$. In fact the transform is an isometric isomorphism of $\mathcal{H}(K)$ onto $\ell^{2}\left(\mathbb{Z}_{+}\right)$.

We turn to the converse: Let $f$ be a fixed function such that $f^{\boldsymbol{\nabla}} \in \ell^{2}\left(\mathbb{Z}_{+}\right)$. Recall (see Theorem 2.2) that

$$
e_{n}(x)=\sum_{j=0}^{n}(-1)^{j+n}\left(\begin{array}{l}
n \\
j
\end{array}\right) K_{j}(x) .
$$

Since the functions $e_{n}$ form an orthonormal basis of $\mathcal{H}(K)$, we conclude that

$$
F:=\sum_{n=0}^{\infty} f^{\mathbf{v}}(n) e_{n} \in \mathcal{H}(K)
$$

and moreover,

$$
\left\langle F, e_{n}\right\rangle_{\mathcal{H}(K)}=f^{\boldsymbol{\nabla}}(n), \quad \forall n \in \mathbb{Z}_{+}
$$

Using 3.25) again, together with the reproducing kernel property for $F$ we conclude that

$$
f(x)=F(x), \quad \forall x \in \mathbb{Z}_{+},
$$

and so $f \in \mathcal{H}(K)$ as claimed. 
Definition 3.8. For $f: \mathbb{Z}_{+} \longrightarrow \mathbb{R}$ we define the following Hurwitz transformation:

$$
f \mapsto H(f)(z)=\sum_{x=0}^{\infty} \frac{f(x)}{x !} z^{x}=e^{z} \sum_{n=0}^{\infty} \frac{f^{\mathbf{v}}(n)}{n !} z^{n},
$$

where $z \in \mathbb{C}$ is a generating function variable.

That the two expressions for $H(f)$ coincide can be checked as follows. For $n=0,1, \ldots$ we have the convolution expression:

$$
\frac{f^{\mathbf{v}}(n) z^{n}}{n !}=\sum_{x=0}^{n} \frac{f(x) z^{x}}{x !} \cdot \frac{(-1)^{n-x} z^{n-x}}{(n-x) !} .
$$

The result follows from the theorem on the coefficients of a product of power series.

We have the following corollary to Corollary 3.7 .

Corollary 3.9. Let $f \in \mathcal{H}(K)$, and consider $f^{\boldsymbol{\nabla}} \in \ell^{2}\left(\mathbb{Z}_{+}\right)$, see (3.24). Then the following hold for the respective Hurwitz-transforms:

$$
H(f)(z)=e^{z} H\left(f^{\nabla}\right)(z) .
$$

In particular, $H(f)$ is an entire function.

The following proposition will be used in Corollary 3.9 .

Proposition 3.10. Assume $(f(x))_{x \in \mathbb{Z}_{+}} \in \ell^{2}\left(\mathbb{Z}_{+}\right)$. Then the Hurwitz transform $H(f)$ is an entire function of $z$.

Proof. Indeed, for any $z \in \mathbb{C}$, the sequence

$$
\left(\frac{z^{x}}{x !}\right)_{x \in \mathbb{Z}_{+}} \in \ell^{2}\left(\mathbb{Z}_{+}\right) .
$$

By the Cauchy-Schwarz inequality, we see that the series (3.28) converges then absolutely for all complex numbers $z$.

Proof. By Corollary 3.7 $f \in \mathcal{H}(K)$ if and only if there exists $\left(a_{n}\right)_{n \in \mathbb{Z}_{+}} \in$ $\ell^{2}\left(\mathbb{Z}_{+}\right)$such that

$$
f=\sum_{n=0}^{\infty} a_{n} e_{n}
$$

with

$$
a_{n}=f^{\mathbf{\nabla}}(n)=\sum_{x=0}^{n}(-1)^{n+x}\left(\begin{array}{l}
n \\
x
\end{array}\right) f(x) .
$$


In a way similar to (3.29) we have:

$$
\frac{f^{\mathbf{\nabla}}(n)}{n !}=\sum_{x=0}^{n} \frac{f(x)}{x !} \frac{(-1)^{n-x}}{(n-x) !} .
$$

By Proposition 3.10, the function $H\left(f^{\mathbf{v}}\right)$ is entire. Since

$$
\frac{f(n)}{n !}=\sum_{x=0}^{n} \frac{f^{\mathbf{\nabla}}(x)}{x !} \frac{1}{(n-x) !},
$$

classical results on power series and convolution implies that

$$
H(f)(z)=\left(H\left(f^{\mathbf{v}}\right)(z)\right) e^{z},
$$

and $H(f)$ is in particular an entire function.

Proposition 3.11. Let $f: \mathbb{Z}_{+} \longrightarrow \mathbb{R}$, and assume that $f \in \mathcal{H}(K)$. Then, for every $\epsilon>0$ there exists $M>0$ such that

$$
|f(n)| \leq M n ! \epsilon^{n}, \quad n \in \mathbb{Z}_{+} .
$$

Proof. From the preceding proposition we know that $H(f)$ is an entire function. Thus for every $r>0$ there exists an $M>0$ such that

$$
r^{n} \frac{|f(n)|}{n !} \leq M, \quad n \in \mathbb{Z}_{+} .
$$

The result follows with $r=1 / \epsilon$.

We note that (3.36) is not sufficient to guarantee that $f \in \mathcal{H}(K)$. Indeed, the series

$$
\sum_{n=0}^{\infty} \frac{f^{\mathbf{v}}(n)}{n !} z^{n}
$$

may define an entire function even when $\left(f^{\boldsymbol{\nabla}}(n)\right)_{n \in \mathbb{Z}_{+}} \notin \ell^{2}\left(\mathbb{Z}_{+}\right)$. For example consider the function $g_{a}$ given by:

$$
g_{a}(y)=\frac{a^{y}}{y !}, \quad y \in \mathbb{Z}_{+} .
$$

Then

$$
\begin{aligned}
g_{a}^{\mathbf{\nabla}}(n) & =(-1)^{n} \sum_{x=0}^{n}\left(\begin{array}{l}
n \\
x
\end{array}\right) \frac{(-a)^{x}}{x !} \\
& =(-1)^{n} \sum_{x=0}^{n}(-a)^{x} \frac{n(n-1) \cdots(n-x+1)}{(x !)^{2}},
\end{aligned}
$$

and so $\left(g_{a}^{\mathbf{v}}(n)\right)_{n \in \mathbb{Z}_{+}} \notin \ell^{2}\left(\mathbb{Z}_{+}\right)$. On the other hand,

$$
H\left(g_{a}^{\mathbf{\nabla}}\right)(z)=e^{-z}\left(H\left(g_{a}\right)(z)\right)=e^{-z} \sum_{n=0}^{\infty} \frac{(a z)^{n}}{(n !)^{2}}
$$

is entire. But $g_{a} \notin \mathcal{H}(K)$ since $\left(g_{a}^{\mathbf{\nabla}}(n)\right)_{n \in \mathbb{Z}_{+}} \notin \ell^{2}\left(\mathbb{Z}_{+}\right)$. 
Corollary 3.12. Let $f: \mathbb{Z}_{+} \longrightarrow \mathbb{R}$ be a function, and assume that $f \in$ $\mathcal{H}(K)$. Then there exists $M<\infty$ such that

$$
|f(x)|^{2} \leq M\left(\begin{array}{c}
2 x \\
x
\end{array}\right), \quad \forall x \in \mathbb{Z}_{+} .
$$

(In other words, condition (3.38) is necessary for $f$ to be in $\mathcal{H}(K)$ ).

Proof. Using the Cauchy-Schwarz inequality we get

$$
\begin{aligned}
|f(x)|^{2} & \leq\|f\|_{\mathcal{H}(K)}^{2} \cdot K(x, x) \\
& =\|f\|_{\mathcal{H}(K)}^{2} \cdot\left(\begin{array}{c}
2 x \\
x
\end{array}\right)
\end{aligned}
$$

See (2.5) for the latter.

Remark 3.13. Let $a \in \mathbb{R} \backslash\{0\}$, and let $g_{a}$ be given by (3.37), that is

$$
g_{a}(x)=\frac{a^{x}}{x !}, \quad x \in \mathbb{Z}_{+},
$$

with as usual $0 !=1$. The condition (3.38) is satisfied, but $g_{a}^{\mathbf{\nabla}}$ is not in $\ell_{2}\left(\mathcal{Z}_{+}\right)$. We conclude that condition (3.38) is not sufficient and $g_{a} \notin \mathcal{H}(K)$.

We now define an isometric isomorphism between the reproducing kernel Hilbert space $\mathcal{H}(K)$ and the reproducing kernel Hilbert space $\mathcal{H}\left(K_{2}\right)$ of entire functions associated to the positive definite function

$$
K_{2}(\zeta, z)=\sum_{n=0}^{\infty} \frac{\zeta^{n} z^{* n}}{(n !)^{2}}, \quad \zeta, z \in \mathbb{C} .
$$

An orthonormal basis of the space $\mathcal{H}\left(K_{2}\right)$ is given by the functions

$$
\zeta \mapsto \frac{\zeta^{n}}{n !}, \quad n=0,1, \ldots
$$

For $f$ a real- or complex- valued function defined on $\mathbb{Z}_{+}$we define the map

$$
(\mathscr{H}(f))(\zeta)=e^{-\zeta}(H(f))(\zeta) .
$$

Theorem 3.14. The map $\mathscr{H}$ is unitary from $\mathcal{H}(K)$ onto $\mathcal{H}\left(K_{2}\right)$, i.e, is isometric and onto.

Proof. Corollary 3.7 implies that $\mathscr{H}$ maps $\mathcal{H}(K)$ into $\mathcal{H}\left(K_{2}\right)$. Indeed $f$ belongs to $\mathcal{H}(K)$ if and only if the transform $f^{\boldsymbol{\nabla}}$ belongs to $\ell^{2}\left(\mathbb{Z}_{+}\right)$. But the function

$$
(\mathscr{H}(f))(\zeta)=e^{-\zeta} \sum_{n=0}^{\infty} f(x) \frac{\zeta^{x}}{x !}=\sum_{n=0}^{\infty} f^{\boldsymbol{\nabla}}(n) \frac{\zeta^{n}}{n !}
$$

belongs to $\mathcal{H}\left(K_{2}\right)$ since the functions (3.41) form an orthonormal basis of $\mathcal{H}\left(K_{2}\right)$ and $f^{\boldsymbol{}} \in \ell^{2}\left(\mathbb{Z}_{+}\right)$. 
To finish the proof, we show that the map $\mathscr{H}$ sends an orthonormal basis of $\mathcal{H}(K)$ onto an orthonormal basis of $\mathcal{H}\left(K_{2}\right)$. We claim that

$$
\left(\mathscr{H}\left(e_{n}\right)\right)(\zeta)=\frac{\zeta^{n}}{n !}, \quad n=0,1, \ldots
$$

Indeed

$$
\begin{aligned}
\left(\mathscr{H}\left(e_{n}\right)\right)(\zeta) & =e^{-\zeta} \sum_{x=n}^{\infty} e_{n}(x) \frac{\zeta^{x}}{x !} \\
& =e^{-\zeta} \frac{\zeta^{n}}{n !} \sum_{x=n}^{\infty} \frac{\zeta^{x-n}}{(x-n) !} \\
& =\frac{\zeta^{n}}{n !}
\end{aligned}
$$

concluding the proof.

The following theorem establishes a co-product for the binomial functions $e_{n}$ in (1.2). It follows from our analysis that the functions $\left\{e_{n} \mid n \in \mathbb{N}_{0}\right\}$ generate a hypergroup $H G$; in the sense of Lasser et al, see [13. Here we refer to our formula (3.47) below for the co-product in $H G$. And in (3.48) we give an explicit formula for the coefficients defining the co-product.

\section{Theorem 3.15.}

(1) It holds that

$$
e_{n}^{\mathbf{\nabla}}(x)=\delta_{n, x}
$$

(2) We have

$$
e_{n} \cdot e_{m}=\sum_{k=m \vee n}^{m+n}\left(e_{m} \cdot e_{n}\right)^{\mathbf{\nabla}}(k) e_{k}
$$

where

$$
\left(e_{m} \cdot e_{n}\right) \mathbf{\nabla}(k)=(-1)^{n+k}\left(\begin{array}{l}
k \\
n
\end{array}\right)\left(\sum_{\ell=0}^{k-n}\left(\begin{array}{c}
k-n \\
\ell
\end{array}\right)\left(\begin{array}{c}
\ell+n \\
m
\end{array}\right)\right) .
$$

For example, we have

$$
e_{1} \cdot e_{n}=n e_{n}+(n+1) e_{n+1} .
$$

Proof of Theorem 3.15. The first claim is a mere rewriting of the second equality in (3.12). To prove (3.48) we assume $m \leq n$ (so that $m \vee n=n$ ) and 
write

$$
\begin{aligned}
\left(e_{m} \cdot e_{n}\right)^{\mathbf{v}}(k) & =\sum_{y=n}^{k}(-1)^{y+k}\left(\begin{array}{l}
k \\
y
\end{array}\right)\left(\begin{array}{c}
y \\
n
\end{array}\right)\left(\begin{array}{c}
y \\
m
\end{array}\right) \\
& =\sum_{y=n}^{k}\left(\begin{array}{c}
k \\
n
\end{array}\right)\left(\begin{array}{c}
k-n \\
y-n
\end{array}\right)\left(\begin{array}{c}
y \\
m
\end{array}\right) \\
& =\left(\begin{array}{l}
k \\
n
\end{array}\right)(-1)^{n+k}\left(\sum_{\ell=0}^{k-n}(-1)^{\ell}\left(\begin{array}{c}
k-n \\
\ell
\end{array}\right)\left(\begin{array}{c}
\ell+n \\
m
\end{array}\right)\right)
\end{aligned}
$$

\section{THE SECOND TRANSFORM}

We show that the two classes of transforms in our study are two sides of a harmonic analysis for the reproducing kernel Hilbert space $\mathcal{H}(K)$, see Theorem 1.1 and Theorem 4.5 below. In the sense of computations, a main distinction between the two is that the first class of transforms involves only finite summations, while the second infinite; see e.g., Definition 4.3, and Proposition 4.6. In the terminology of the infinite square matrices in Sections 1 and 7 the distinction reflects the difference in the algebra of infinite lower triangular matrices, vs upper triangular ones. In Theorem 4.8, we establish explicit inversion formulas. Caution, while the range of the respective isometric transforms are Hilbert spaces, they differ from one to the other.

We note that the function

$$
(-1)^{y+z} K(y, z)=(-1)^{y}(-1)^{z} K(y, z)
$$

is still positive on $\mathbb{Z}_{+}$, and recall that we denote by $\ell_{0}\left(\mathbb{Z}_{+}\right)$the vector space of sequences from $\mathbb{Z}_{+}$into $\mathbb{R}$, with compact support. We endow $\ell_{0}\left(\mathbb{Z}_{+}\right)$with the bilinear form

$$
\langle f, g\rangle_{K}=\sum_{x=0}^{\infty} \sum_{y=0}^{\infty}(-1)^{y+z} f(x) g(y) K(x, y) .
$$

Proposition 4.1. The space $\ell_{0}\left(\mathbb{Z}_{+}\right)$endowed with the bilinear form (4.2) is a pre-Hilbert space.

Proof. Bilinearity of $\langle\cdot, \cdot\rangle_{K}$ is clear from the definition, while the positivity property

$$
\langle f, f\rangle_{K} \geq 0, \quad \forall f \in \ell_{0}\left(\mathbb{Z}_{+}\right)
$$

follows from the fact that the function (4.1) is positive definite on $\mathbb{Z}_{+}$. We now show that the form $\langle\cdot, \cdot\rangle_{K}$ is non-degenerate. Let $g \in \ell_{0}\left(\mathbb{Z}_{+}\right)$be such 
that

$$
\langle f, g\rangle_{K}=0, \quad \forall f \in \ell_{0}\left(\mathbb{Z}_{+}\right)
$$

In particular,

$$
\langle g, g\rangle_{K}=0 .
$$

This cannot be since all the submatrices built from the kernel $K$ from different points are strictly positive (see Theorem 1.5 ).

We denote by $\ell_{K}^{2}\left(\mathbb{Z}_{+}\right)$the closure of $\ell_{0}\left(\mathbb{Z}_{+}\right)$with respect to $\langle\cdot, \cdot\rangle_{K}$.

Proposition 4.2. The map

$$
f \mapsto \sum_{y=0}^{\infty} K(x, y)(-1)^{y} f(y),
$$

first defined for $f \in \ell_{0}\left(\mathbb{Z}_{+}\right)$, extends to a unitary map from $\ell_{K}^{2}\left(\mathbb{Z}_{+}\right)$onto $\mathcal{H}(K)$.

Proof. Let $f \in \ell_{0}\left(\mathbb{Z}_{+}\right)$.We have (recall that all the sums are finite since $f$ has finite support)

$$
\begin{aligned}
\langle f, f\rangle_{K} & =\sum_{n, m=0}^{\infty}(-1)^{n}(-1)^{m} f(n) f(m) K(n, m) \\
& =\left\langle\sum_{n=0}^{\infty} K_{n}(-1)^{n} f(n), \sum_{m=0}^{\infty} K_{m}(-1)^{m} f(m)\right\rangle_{\mathcal{H}(K)} .
\end{aligned}
$$

The claim then follows from density and from the continuity of the inner product.

Definition 4.3. Let $f \in \ell_{0}\left(\mathbb{Z}_{+}\right)$. We set

$$
f^{\Delta}(x)=\sum_{y=x}^{\infty}(-1)^{x+y}\left(\begin{array}{l}
y \\
x
\end{array}\right) f(y) .
$$

We note that (4.8) can be rewritten as

$$
f^{\Delta}=M(-1) f,
$$

where $M(\lambda)$ is defined by (7.12).

Explicitly, the series is written as

$$
\begin{gathered}
f^{\Delta}(x)=(-1)^{x}\left(f(x)-(x+1) f(x+1)+\frac{(x+2)(x+1)}{2} f(x+2)-\right. \\
\left.-\frac{(x+3)(x+2)(x+1)}{6} f(x+3)+\cdots\right)
\end{gathered}
$$


Note that $f^{\Delta} \in \ell_{0}\left(\mathbb{Z}_{+}\right)$, and more precisely, if $f(x)=0$ for $x \geq N$ then $f^{\Delta}(x)=0$ for $x \geq N$.

Example 4.4. Let $g_{a}$ be defined by (3.37): $g_{a}(y)=\frac{a^{y}}{y !}$, where $a \in \mathbb{R}$. Then,

$$
g_{a}^{\Delta}=e^{-a} g_{a}
$$

Indeed,

$$
\begin{aligned}
g_{a}^{\Delta}(x) & =\sum_{y=x}^{\infty}(-1)^{x+y}\left(\begin{array}{c}
y \\
x
\end{array}\right) \frac{a^{y}}{y !} \\
& =\sum_{y=x}^{\infty}(-1)^{x+y} \frac{a^{y}}{x !(y-x) !} \\
& =\frac{1}{x !} \sum_{y=x}^{\infty}(-1)^{x+y} \frac{a^{y}}{(y-x) !} \\
& =\frac{a^{x}}{x !} \sum_{u=0}^{\infty}(-1)^{u} \frac{a^{u}}{u !} \\
& =e^{-a} \frac{a^{x}}{x !} .
\end{aligned}
$$

where we have used the change of index $y-x=u$ to go from the third to the fourth line.

We note the following: though $g_{a}$ does not belong to $\mathcal{H}(K)$ unless $a=0$, the $\Delta$ transform of $g_{a}$ exists for all real $a$.

In preparation of Theorem 4.5 we introduce the notation $s(f)$ as:

$$
f \quad \mapsto \quad s(f):=\sum_{x \in \mathbb{Z}_{+}} K_{x} f(x)
$$

Theorem 4.5. The map $f \mapsto s\left(f^{\Delta}\right)$ extends to a unitary map from $\mathcal{H}(K)$ onto $\ell^{2}\left(\mathbb{Z}_{+}\right)$. 
Proof. Let $f \in \ell_{0}\left(\mathbb{Z}_{+}\right)$. We have (recall that all the sums are finite since $f$ has finite support):

$$
\begin{aligned}
\left\langle s\left(f^{\Delta}\right), s\left(f^{\Delta}\right)\right\rangle_{\mathcal{H}(K)}= & \sum_{x_{1}=0}^{\infty} \sum_{x_{2}=0}^{\infty} s\left(f^{\Delta}\right)\left(x_{1}\right) s\left(f^{\Delta}\right)\left(x_{2}\right) K\left(x_{1}, x_{2}\right) \\
= & \sum_{x_{1}=0}^{\infty} \sum_{x_{2}=0}^{\infty}\left(\sum_{y=x_{1}}^{\infty}(-1)^{x_{1}+y}\left(\begin{array}{c}
y \\
x_{1}
\end{array}\right) f(y)\right) \times \\
& \times K\left(x_{1}, x_{2}\right)\left(\sum_{z=x_{2}}^{\infty}(-1)^{x_{2}+z}\left(\begin{array}{c}
z \\
x_{2}
\end{array}\right) f(z)\right) \\
= & \sum_{x_{1}=0}^{\infty} \sum_{x_{2}=0}^{\infty} \sum_{y=x_{1}}^{\infty} \sum_{z=x_{2}}^{\infty} \sum_{j=0}^{x_{1} \wedge x_{2}}(-1)^{x_{1}+x_{2}+y+z} f(y) f(z) \times \\
= & \sum_{y=0}^{\infty} \sum_{z=0}^{\infty} f(y) f(z)\left(\begin{array}{c}
y \\
x_{1}
\end{array}\right)\left(\begin{array}{c}
z \\
x_{2}
\end{array}\right)\left(\begin{array}{c}
x_{1} \\
j
\end{array}\right)\left(\begin{array}{c}
x_{2} \\
j
\end{array}\right) \\
\times & \left.\times\left(\begin{array}{c}
y \\
j \\
\sum^{y}=0 \\
x_{1}=j
\end{array}\right)^{x_{1}+y}\left(\begin{array}{c}
y \\
x_{1}
\end{array}\right)\left(\begin{array}{c}
x_{1} \\
j
\end{array}\right)\right) \times \\
x_{2}=j &
\end{aligned}
$$

and using Lemma 2.1

$$
\begin{aligned}
& =\sum_{j=0}^{\infty} \sum_{y=0}^{\infty} \sum_{z=0}^{\infty} f(y) f(z) \delta_{j, y} \delta_{j, z} \\
& =\sum_{j=0}^{\infty} f(j)^{2}
\end{aligned}
$$

We set $\mathscr{A}(f)=s\left(f^{\Delta}\right)$. It turns out (see the next proposition), that although $s(f)$ is difficult to compute for general value of $f$, the operator $\mathcal{A}$ and its adjoint are easier to handle.

Proposition 4.6. The adjoint $\mathscr{A}^{*}$ of the operator $\mathscr{A}$ is given by:

$$
\left(\mathscr{A}^{*}(\varphi)\right)(x)=\sum_{y=x}^{\infty}(-1)^{x+y}\left(\begin{array}{l}
y \\
x
\end{array}\right) \varphi(y), \quad \varphi \in \mathcal{H}(K),
$$

and with values in $\ell^{2}\left(\mathbb{Z}_{+}\right)$. 
Proof. We check the result for $\varphi_{K_{y_{0}}}$. The general case follows then by continuity. Let $f \in \ell^{2}\left(\mathbb{Z}_{+}\right)$. We have:

$$
\begin{aligned}
\langle\mathscr{A} f, \varphi\rangle_{\mathcal{H}(K)} & =(\mathscr{A} f)\left(y_{0}\right) \\
& =\sum_{t=0}^{\infty} f^{\Delta}(t) K\left(y_{0}, t\right) \\
& =\sum_{t=0}^{\infty} \sum_{s=t}^{\infty}(-1)^{t+s} f(s)\left(\begin{array}{l}
s \\
t
\end{array}\right) K\left(y_{0}, t\right) \\
& =\sum_{s=0}^{\infty} f(s)(-1)^{s} \sum_{t=0}^{s}\left(\begin{array}{l}
s \\
t
\end{array}\right) K\left(y_{0}, t\right),
\end{aligned}
$$

and hence the result.

Definition 4.7. Let $g \in \ell_{0}\left(\mathbb{Z}_{+}\right)$. We set

$$
g^{\nabla}(n)=\sum_{x=n}^{\infty}\left(\begin{array}{l}
x \\
n
\end{array}\right) g(x) .
$$

Theorem 4.8. The map $g \mapsto g \nabla$ extends to a unitary map from $\ell_{K}^{2}\left(\mathbb{Z}_{+}\right)$onto $\ell^{2}\left(\mathbb{Z}_{+}\right)$, and its adjoint is the map $f \mapsto f^{\Delta}$. In particular:

$$
\begin{array}{ll}
\left(g^{\nabla}\right)^{\Delta}=g, & \forall g \in \ell_{K}^{2}\left(\mathbb{Z}_{+}\right) \\
\left(f^{\Delta}\right)^{\nabla}=f, & \forall f \in \ell^{2}\left(\mathbb{Z}_{+}\right) .
\end{array}
$$

Proof. We divide the proof in two steps.

STEP 1: We first prove that the two transforms are inverse of each other. Then we show that they are adjoint of each other.

Let $g \in \ell_{0}\left(\mathbb{Z}_{+}\right)$. We have:

$$
\begin{aligned}
\left(g^{\nabla}\right)^{\Delta}(y) & =\sum_{n=0}^{\infty}(-1)^{n+y}\left(\begin{array}{l}
n \\
y
\end{array}\right)\left(g^{\Delta}(n)\right) \\
& =\sum_{n=y}^{\infty}(-1)^{n+y}\left(\begin{array}{l}
n \\
y
\end{array}\right)\left(\sum_{x=n}^{\infty}\left(\begin{array}{l}
x \\
n
\end{array}\right) g(x)\right) \\
& =\sum_{x=y}^{\infty}\left(\sum_{n=y}^{x}(-1)^{n+y}\left(\begin{array}{l}
n \\
y
\end{array}\right)\left(\begin{array}{l}
x \\
n
\end{array}\right)\right) \\
& =g(y)
\end{aligned}
$$

since (see Lemma 2.1)

$$
\sum_{n=y}^{x}(-1)^{n+y}\left(\begin{array}{l}
n \\
y
\end{array}\right)\left(\begin{array}{l}
x \\
n
\end{array}\right)=\delta_{x, y} .
$$


Similarly, we have for $f \in \ell^{2}\left(\mathbb{Z}_{+}\right)$and $n \geq 0$ :

$$
\begin{aligned}
\left(\left(f^{\Delta}\right)^{\nabla}\right)(n) & =\sum_{x=n}^{\infty}\left(\begin{array}{l}
x \\
n
\end{array}\right) f^{\Delta}(x) \\
& =\sum_{x=n}^{\infty}\left(\begin{array}{l}
x \\
n
\end{array}\right)\left(\sum_{m=x}^{\infty}\left(\begin{array}{c}
m \\
x
\end{array}\right) f(m)\right) \\
& =\sum_{m=n}^{\infty} f(n)\left(\sum_{x=n}^{m}(-1)^{x+m}\left(\begin{array}{c}
m \\
x
\end{array}\right)\left(\begin{array}{l}
x \\
n
\end{array}\right)\right) \\
& =f(m)
\end{aligned}
$$

since (see Lemma 2.1)

$$
\sum_{x=n}^{m}(-1)^{x+m}\left(\begin{array}{c}
m \\
x
\end{array}\right)\left(\begin{array}{l}
x \\
n
\end{array}\right)=\delta_{m, n} .
$$

STEP 2: We now prove that the two transforms are adjoint of each other.

Let $f \in \ell^{2}\left(\mathbb{Z}_{+}\right)$and $g \in \ell_{K}^{2}\left(\mathbb{Z}_{+}\right)$. We have:

$$
\begin{aligned}
\left\langle g, f^{\Delta}\right\rangle_{\ell_{K}^{2}\left(\mathbb{Z}_{+}\right)} & =\sum_{x, y=0}^{\infty} g(x) f^{\Delta}(y) K(x, y) \\
& =\sum_{x, y=0}^{\infty} g(x)\left(\sum_{t=y}^{\infty}(-1)^{t+y} f(t)\left(\begin{array}{l}
t \\
y
\end{array}\right)\right) K(x, y) \\
& =\sum_{t=0}^{\infty} f(t)\left(\sum_{x=0}^{\infty} g(x) \sum_{y=0}^{t}(-1)^{t+y}\left(\begin{array}{l}
t \\
y
\end{array}\right)\right) K(x, y) \\
& =\sum_{t=0}^{\infty} f(t)\left(\sum_{x=t}^{\infty} g(x)\left(\begin{array}{l}
x \\
t
\end{array}\right)\right)
\end{aligned}
$$

where we use (2.4)

$$
=\left\langle g^{\nabla} f\right\rangle_{\ell^{2}\left(\mathbb{Z}_{+}\right)}
$$

To conclude we now prove that the $\nabla$ transform is an isometry. We first prove this on the space $\mathcal{H}_{0}$ of functions from $\mathbb{Z}_{+}$into $\mathbb{R}$ with finite support. 
For $g \in \ell_{0}$ we have:

$$
\begin{aligned}
\left\|g^{\Delta}\right\|_{\mathcal{H}(K)}^{2} & =\sum_{n=0}^{\infty}\left(\sum_{x=n}^{\infty}\left(\begin{array}{l}
x \\
n
\end{array}\right) g(x)\right)^{2} \\
& =\sum_{x, y=0}^{\infty} g(x) g(y)\left(\sum_{n=0}^{x \wedge y}\left(\begin{array}{l}
x \\
n
\end{array}\right)\left(\begin{array}{l}
y \\
n
\end{array}\right)\right) \\
& =\sum_{x, y=0}^{\infty} g(x) g(y) K(x, y) \\
& =\|g\|_{\ell^{2}(K)}^{2} .
\end{aligned}
$$

\section{Motivation From Discrete ANALYTIC FUnCtions}

Here we offer a brief outline of the significance of our binomial functions in the study of discrete analytic functions. While our binomial functions are functions of a single discrete variable (here $\mathbb{Z}_{+}$), we show in [2] that the binomial functions extend uniquely to discrete analytic functions on the $2 D$ lattice, so formulas obtained in the earlier part of our paper have direct implications for Hilbert spaces of discrete analytic functions.

Recall first that a function $f: \mathbb{Z}^{2} \longrightarrow \mathbb{C}$ is said to be discrete analytic if

$$
\forall(x, y) \in \mathbb{Z}^{2}, \quad \frac{f(x+1, y+1)-f(x, y)}{1+i}=\frac{f(x+1, y)-f(x, y+1)}{1-i} .
$$

See [7, 6].

Given a function $f_{0}: \mathbb{Z} \longrightarrow \mathbb{C}$ there are infinitely discrete analytic functions $f$ on $\mathbb{Z}^{2}$ such that $f(x, 0)=f_{0}(x)$. However, when $f_{0}$ is a polynomial, only one of these discrete analytic extensions will be a polynomial in $x, y$. See [6] (we gave a new proof of this fact in [2]). Thus, there exists a unique discrete analytic polynomial $\zeta_{n}(x, y)$ determined by $\zeta_{n}(x, 0) \equiv x^{[n]}$, where $x^{[n]}$ has been defined in (1.7).

\section{The QuAntum CASE}

In a number of questions from mathematical physics (see [10, 9, 11] and the papers cited there), it is of interest to consider the questions from sections 2 through 3 for a deformation family of the binomial functions (1.2). Traditionally the complex deformation parameter is denoted $q$, and the $q$-versions of the binomial formulas were first studied in [4]. The purpose of this section is to prove an analogue of the orthonormal basis theorems but adapted to the 
$q$-binomials.

The $q$-deformed binomial coefficients were defined by Carlitz; see [5, 4]. We fix $q \in \mathbb{R} \backslash\{1\}$, and set

$$
\begin{aligned}
{[x] } & :=\frac{q^{x}-1}{q-1}, \\
{[x]_{n} } & :=[x][x-1] \cdots[x-n+1], \\
{[n] ! } & :=[n]_{n}, \quad \text { and }[0] !=[0]=1, \\
{\left[\begin{array}{l}
x \\
n
\end{array}\right] } & :=\frac{[x]_{n}}{[n] !},
\end{aligned}
$$

where all the expressions depend on the value of $q$. To lighten the notation, we do not stress the $q$-dependence. We introduce

$$
e_{n}(x)= \begin{cases}0, & \text { if } \quad x<n, \\
{\left[\begin{array}{l}
x \\
n
\end{array}\right]=\frac{[x]_{n}}{[n] !},} & \text { if } x \geq n,\end{cases}
$$

and

$$
K(x, y)=\sum_{n=0}^{\infty} e_{n}(x) e_{n}(y), \quad x, y \in \mathbb{Z}_{+} .
$$

The sum (6.3) is always finite and well defined, and defines a positive definite function on $\mathbb{Z}_{+}$. It can also be rewritten as:

$$
K(x, y)=\sum_{n=0}^{x \wedge y}\left[\begin{array}{l}
x \\
n
\end{array}\right]\left[\begin{array}{l}
y \\
n
\end{array}\right] .
$$

The functions $e_{0}, e_{1}, \ldots$ are easily seen to be linearly independent, and so we have:

Theorem 6.1. The functions $e_{0}, e_{1}, \ldots$ form an orthonormal basis of $\mathcal{H}(K)$.

\section{A ONE PARAMETER GROUP OF TRANSFORMS}

Let $\lambda \in(0, \infty)$. The function

$$
K_{\lambda}(x, y)=\sum_{n=0}^{x \wedge y} \lambda^{x+y-2 n}\left(\begin{array}{l}
x \\
n
\end{array}\right)\left(\begin{array}{l}
y \\
n
\end{array}\right)
$$

is positive definite in $\mathbb{Z}_{+}$, and we denote by $\mathcal{H}\left(K_{\lambda}\right)$ the associated reproducing kernel Hilbert space. The functions

$$
e_{n}^{(\lambda)}(x)=\lambda^{x-n} e_{n}(x), \quad, n=0,1, \ldots
$$


form an orthonormal basis of the associated reproducing kernel Hilbert space $\mathcal{H}\left(K_{\lambda}\right)$ Note that the case $\lambda=1$ corresponds to formulas (1.5) and (1.2). Let $L(\lambda)$ denote the Zadeh-transform of the lower triangular infinite matrix $L$ given by (1.9), that is the lower triangular infinite matrix $M(\lambda)$ as follows: For a point $(x, y) \in \mathbb{Z}_{+} \times \mathbb{Z}_{+}$, let $x$ denote row-index and $y$ denote column-index. The matrix $L(\lambda)$ is defined by:

$$
(L(\lambda))_{(x, y)}= \begin{cases}\lambda^{x-y}\left(\begin{array}{l}
x \\
y
\end{array}\right), & \text { if } \quad 0 \leq y \leq x \\
0, & \text { otherwise }\end{cases}
$$

Thus, $L(\lambda)$ is given by

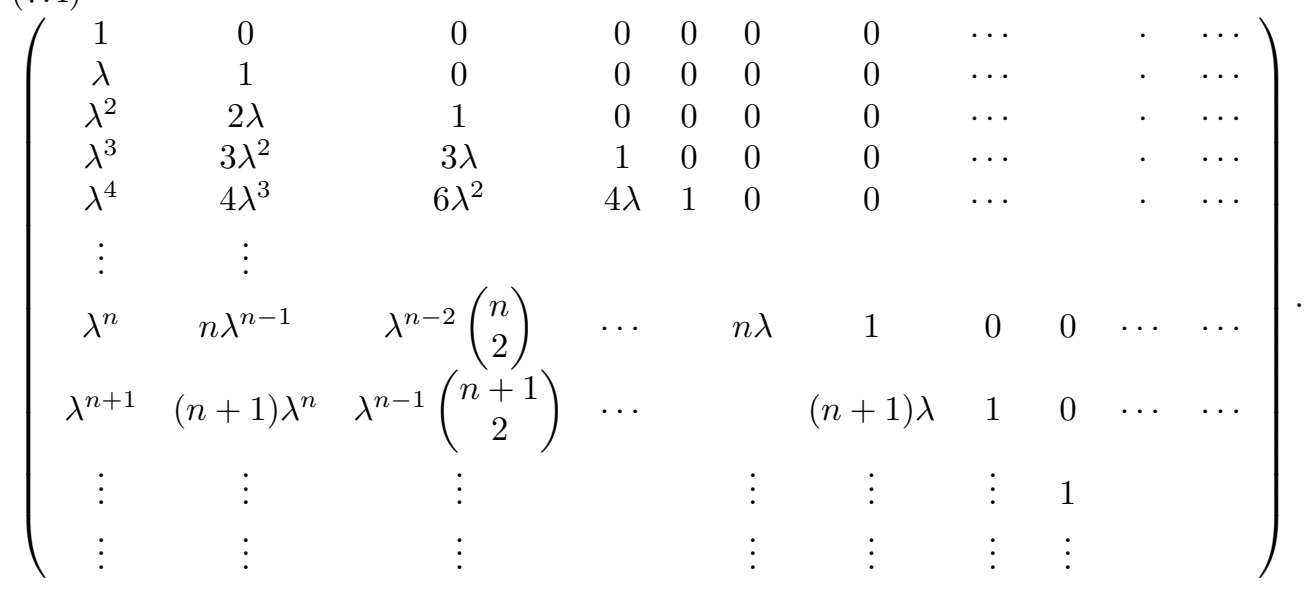

Then the matrix product $L(\lambda) L(\mu)$ makes sense for all $\lambda, \mu \in \mathbb{C}$, with

$$
(L(\lambda) L(\mu))_{(x, y)}=\sum_{k=y}^{x} L(\lambda)_{(x, k)} L(\mu)_{(k, y)}, \quad \lambda, \mu \in \mathbb{C} .
$$

Lemma 7.1. With the notation above it holds that

$$
L(\lambda+\mu)=L(\lambda) L(\mu), \quad \lambda, \mu \in \mathbb{C},
$$

i.e. the matrices in (7.3) define a one-parameter semi-group of infinite matrices.

Proof. We give the details, but note that the computation of (17.5), is essential the same as that given in the proof of Lemma 2.1 above. In other words, when the product in (7.5) is computed, the group law (7.6) follows. More precisely,

$$
\begin{aligned}
L(\lambda) L(\mu) & =D(\lambda) L(1) D(\lambda)^{-1} D(\mu) L(1) D(\mu)^{-1} \\
& =D(\lambda) L(1) D\left(\frac{\mu}{\lambda}\right) L(1) D(\mu)^{-1}
\end{aligned}
$$


But for $y \leq x$ we have:

$$
\left(L(1) D\left(\frac{\mu}{\lambda}\right) L(1)\right)_{(x, y)}=\sum_{k=y}^{x}\left(\begin{array}{l}
x \\
k
\end{array}\right)\left(\frac{\mu}{\lambda}\right)^{k}\left(\begin{array}{l}
k \\
y
\end{array}\right)
$$

and using $\left(\begin{array}{l}x \\ k\end{array}\right)\left(\begin{array}{l}k \\ y\end{array}\right)=\left(\begin{array}{l}x \\ y\end{array}\right)\left(\begin{array}{l}x-y \\ k-y\end{array}\right)$, this equality is equal to

$$
\begin{aligned}
& =\left(\begin{array}{l}
x \\
y
\end{array}\right)\left(\sum_{k=y}^{x}\left(\frac{\mu}{\lambda}\right)^{k}\left(\begin{array}{l}
x-y \\
k-y
\end{array}\right)\right) \\
& =\left(1+\frac{\mu}{\lambda}\right)^{x-y}\left(\frac{\mu}{\lambda}\right)^{y}\left(\begin{array}{l}
x \\
y
\end{array}\right) .
\end{aligned}
$$

Thus

$$
L(1) D\left(\frac{\mu}{\lambda}\right) L(1)=D\left(1+\frac{\mu}{\lambda}\right) L(1) D\left(\frac{\mu}{\mu+\lambda}\right),
$$

and this, together with (7.7) leads to the result.

Corollary 7.2. The one-parameter group $\lambda \mapsto L(\lambda)$ of infinite matrices in (17.4) has the form

$$
L(\lambda)=\exp (\lambda A),
$$

where $A$ is the following sub-diagonal banded matrix

$$
A_{(x, y)}=x \delta_{0}(x-y-1)
$$

see (7.11).

$$
\left(\begin{array}{ccccccccccc}
0 & 0 & 0 & 0 & 0 & 0 & 0 & \cdots & & . & \cdots \\
1 & 0 & 0 & 0 & 0 & 0 & 0 & \cdots & & . & \cdots \\
0 & 2 & 0 & 0 & 0 & 0 & 0 & \cdots & & . & \cdots \\
0 & 0 & 3 & 0 & 0 & 0 & 0 & \cdots & & . & \cdots \\
\vdots & \vdots & & & & & & & & & \\
0 & 0 & \ldots & \ldots & & n-1 & 0 & 0 & 0 & \cdots & \cdots \\
0 & 0 & 0 & \cdots & & & n & 0 & 0 & \cdots & \cdots \\
0 & 0 & \cdots & \cdots & 0 & 0 & n+1 & 0 & \cdots & \cdots \\
\vdots & \vdots & \vdots & & \vdots & \vdots & \vdots & \vdots & &
\end{array}\right) .
$$

We denote

$$
M(\lambda)=L(\lambda)^{*} .
$$

We define

$$
\left(T_{-\lambda}\left(e_{n}^{(\lambda)}\right)\right)(x)=\delta_{n, x}
$$


The proof of the following theorem is easy and will be omitted.

Theorem 7.3. The map

$$
T_{-\lambda}: f \mapsto L(\lambda)^{*} f
$$

is an isometry from $\mathcal{H}\left(K_{\lambda}\right)$ into $\ell^{2}\left(\mathbb{Z}_{+}\right)$.

As a corollary we have:

$$
\left(L(\lambda) L(\lambda)^{*}\right)_{(x, y)}=K_{\lambda}(x, y)=\sum_{n=0}^{x \wedge y} e_{n}^{(\lambda)}(x) e_{n}^{(\lambda)}(y)^{*}
$$

where $e_{n}^{(\lambda)}(x)=\lambda^{x-n} e_{n}(x)$.

Before stating another corollary to this theorem we mention the following lemma, whose proof is left to the reader.

Lemma 7.4. Let $\left(X_{x}(\cdot)\right)$ be a Gaussian process with index $x$, with mean zero and probability space $(\Omega, \mathcal{F}, P)$. Let $f$ be a non vanishing function on the index set. Then, the Gaussian process $\left(Y_{x}(\cdot)\right)=\left(f(x) X_{x}(\cdot)\right)$ has the same probability space but with covariance $\mathbb{E}\left(Y_{x} Y_{y}\right)=f(x) \mathbb{E}\left(X_{x} X_{y}\right) f(y)$.

Corollary 7.5. Let $\left(\Omega, \mathcal{F}, P^{\lambda}\right)$ be probability spaces and associated stochastic processes $X^{(\lambda)}$ such that

$$
\mathbb{E}_{\lambda}\left(X_{x}^{(\lambda)} X_{y}^{(\lambda)}\right)=K_{\lambda}(x, y)
$$

Then the probability measures $P^{\lambda}$ are mutually singular, as we vary $\lambda \in$ $(0, \infty)$.

Proof. By the above lemma, the process $Y_{x}^{(\lambda)}=\frac{1}{\lambda^{x}} X_{x}^{(\lambda)}$ has the same probability space but covariance function

$$
\mathbb{E}_{\lambda}\left(Y_{x}^{(\lambda)} Y_{y}^{(\lambda)}\right)=\frac{1}{\lambda^{x} \lambda^{y}} K_{\lambda}(x, y) .
$$

We write for $\lambda_{1}$ and $\lambda_{2} \in(0, \infty)$

$$
\begin{aligned}
\frac{1}{\lambda_{1}^{x} \lambda_{1}^{y}} K_{\lambda_{1}}(x, y) & =\sum_{n=0}^{\infty} \lambda_{1}^{-2 n} e_{n}(x) e_{n}(y) \\
\frac{1}{\lambda_{2}^{x} \lambda_{2}^{y}} K_{\lambda_{2}}(x, y) & =\sum_{n=0}^{\infty} \lambda_{2}^{-2 n} e_{n}(x) e_{n}(y) \\
& =\sum_{n=0}^{\infty} \mu_{n} \lambda_{1}^{-2 n} e_{n}(x) e_{n}(y),
\end{aligned}
$$


where we have set $\mu_{n}=\frac{\lambda_{2}^{2 n}}{\lambda_{1}^{2 n}}$. By [12, Theorems 4.3 and 4.4, p.27], we see that $P^{\lambda_{1}}$ and $P^{\lambda_{2}}$ are equivalent if and only if

$$
\sum_{n=0}^{\infty}\left(1-\mu_{n}\right)^{2}<\infty
$$

which holds if and only if $\lambda_{1}=\lambda_{2}$.

Acknowledgments: It is a pleasure to thank the referee for his/her thorough reading of the paper and very useful comments.

\section{REFERENCES}

1. D. Alpay. The Schur algorithm, reproducing kernel spaces and system theory. American Mathematical Society, Providence, RI, 2001. Translated from the 1998 French original by Stephen S. Wilson, Panoramas et Synthèses.

2. D. Alpay, P. Jorgensen, R. Seager, and D. Volok. On discrete analytic functions: Products, rational functions and reproducing kernels. Journal of Applied Mathematics and Computing, 41:393-426, 2013.

3. N. Aronszajn. Theory of reproducing kernels. Trans. Amer. Math. Soc., 68:337-404, 1950.

4. L. Carlitz. $q$-Bernoulli numbers and polynomials. Duke Math. J., 15:987-1000, 1948.

5. L. Carlitz. Expansions of $q$-Bernoulli numbers. Duke Math. J., 25:355-364, 1958.

6. R. J. Duffin. Basic properties of discrete analytic functions. Duke Math. J., 23:335-363, 1956.

7. Jacqueline Ferrand. Fonctions préharmoniques et fonctions préholomorphes. Bull. Sci. Math. (2), 68:152-180, 1944.

8. A. Hurwitz. Ueber die Entwickelungscoefficienten der lemniscatischen Functionen. Math. Ann., 51(2):196-226, 1898.

9. P. E. T. Jorgensen and A. M. Paolucci. States on the Cuntz algebras and $p$-adic random walks. J. Aust. Math. Soc., 90(2):197-211, 2011.

10. P. E. T. Jorgensen and A. M. Paolucci. Markov measures and extended zeta functions. J. Appl. Math. Comput., 38(1-2):305-323, 2012.

11. P. E. T. Jorgensen, L. M. Schmitt, and R. F. Werner. q-relations and stability of $C^{*}$-isomorphism classes. In Algebraic methods in operator theory, pages 261-271. Birkhäuser Boston, Boston, MA, 1994.

12. Ole Groth Jørsboe. Equivalence or singularity of Gaussian measures on function spaces. Various Publications Series, No. 4. Matematisk Institut, Aarhus Universitet, Aarhus, 1968.

13. Rupert Lasser and Eva Perreiter. Homomorphisms of $l^{1}$-algebras on signed polynomial hypergroups. Banach J. Math. Anal., 4(2):1-10, 2010.

14. L. Mendo. Asymptotically optimum estimation of a probability in inverse binomial sampling under general loss functions. J. Statist. Plann. Inference, 142(10):2862-2870, 2012.

15. Luis Mendo and José M. Hernando. Estimation of a probability with optimum guaranteed confidence in inverse binomial sampling. Bernoulli, 16(2):493-513, 2010.

16. H. Meschkovski. Hilbertsche Räume mit Kernfunktion. Springer-Verlag, 1962.

17. G. Polya and G. Szegö. Problems and Theorems in Analysis II. Theory of functions, zeros, polynomials, determinants, number theory, geometry. Springer, 1998. First published in 1924 as vol. 193 of the Grundlehren der mathematiken Wissenschaften. 
18. S. Saitoh. Theory of reproducing kernels and its applications, volume 189. Longman scientific and technical, 1988.

19. L. Schwartz. Sous espaces hilbertiens d'espaces vectoriels topologiques et noyaux associés (noyaux reproduisants). J. Analyse Math., 13:115-256, 1964.

20. A. Tucker Applied Combinatorics (third edition) John Wyley and Sons, 1995.

21. Wojciech Zieliński. The shortest Clopper-Pearson confidence interval for binomial probability. Comm. Statist. Simulation Comput., 39(1):188-193, 2010.

(DA) Department of Mathematics

Ben Gurion University of the Negev

P.O.B. 653,

BE'ER SHEVA 84105,

ISRAEL

E-mail address: dany@math.bgu.ac.il

(PJ) Department of Mathematics $14 \mathrm{MLH}$

The University of Iowa, Iowa City,

IA 52242-1419 USA

E-mail address: jorgen@math.uiowa.edu 